\title{
IRRELEVANT SOUND
}

The Irrelevant Sound Effect: What Needs Modelling, and a Tentative Model.

\section{M.P.A. Page,}

Department of Psychology, University of Hertfordshire.

\author{
D.G. Norris, \\ MRC Cognition and Brain Sciences Unit, Cambridge, UK.
}




\begin{abstract}
This paper reviews the literature on the irrelevant sound effect and concludes that, contrary to some claims, the data consistently show that irrelevant sound and articulatory suppression are not functionally equivalent. We evaluate the contribution of Larsen and Baddeley (in press) and briefly discuss additional data in support of their position. We perform an error analysis on data from their third experiment and simulate detailed aspects of those data using our primacy model of immediate serial recall. Our model is briefly related to a number of findings in the literature on irrelevant sound.
\end{abstract}


Introduction

In two important papers (Larsen, Baddeley \& Andrade, 2000; Larsen \& Baddeley, in press), Larsen and colleagues have gone a considerable way towards clarifying the nature of the irrelevant sound effect and its implications for theories of short-term memory. In this paper, we extend the discussion in several directions. First, we briefly review the literature relevant to a comparison between the effect of irrelevant sound and that of articulatory suppression. In particular we will focus on the interactions of both with the phonological similarity effect, as observed in the serial recall of visually presented materials. Superficially, the data appear somewhat inconsistent. Some experiments show that irrelevant sound eliminates the phonological similarity effect, just as articulatory suppression is known to do (Estes, 1973; Levi, 1971; Murray, 1968; etc.) — these results have been taken as evidence against the working memory model (Baddeley, 1986; Baddeley \& Hitch, 1974). Other experiments, by contrast, show reliable effects of phonological similarity in the presence of irrelevant sound. Our review will show that the data from different studies are actually much more consistent than is often thought, and that they sit comfortably within the working memory framework once differences in experimental procedure are taken into account. In the second part of the paper, we will present a detailed analysis of Larsen and Baddeley's data and show how these data can be simulated by the primacy model of immediate serial recall (Page \& Norris, 1998). We will relate this tentative model to a broad range of data on the irrelevant sound effect.

Are irrelevant sound and articulatory suppression equivalent? 
Colle and Welsh (1976) were first to show that irrelevant sound impaired serial recall of visually presented information from short-term memory. In their study, irrelevant sound was played throughout presentation, retention, and recall of lists of eight visually presented letters. Half of the lists were made up of letters having phonologically different letter-names, the other half of phonologically similar (rhyming) letter-names. Colle and Welsh found a reliable interaction between phonological confusability and irrelevant sound: there was a reliable effect of irrelevant sound only for the phonologically-different letter-names; and there was no effect of phonological similarity in the presence of irrelevant sound. This early result set the scene for several later claims that irrelevant sound and articulatory suppression were functionally equivalent, given that both apparently eliminated the effect of phonological similarity.

Salamé and Baddeley $(1982 ; 1986)$ followed up this work and offered evidence that the situation was rather more complex. Specifically, Salamé and Baddeley (1986) showed that one could find additive effects of phonological similarity and irrelevant sound, provided list length was not sufficiently long to discourage subjects from using what Baddeley and Hitch (1974) had called the phonological loop. Salamé and Baddeley found additive effects at list lengths five, six and seven, but found no effect of phonological similarity, together with a preserved effect of irrelevant sound, at list length eight. This clearly qualified any conclusions to be drawn from Colle and Welsh (1976 - who had used eight-item lists), given that, for Salamé and Baddeley, irrelevant sound had proved unable to abolish the phonological similarity effect at three of the four list-lengths tested.

Jones and Macken (1995) disconfirmed Salamé and Baddeley's (1982) 
hypothesis that the phonological characteristics of the irrelevant sound interfered directly with the contents of the phonological store. In their fourth experiment, Jones \& Macken (1995, p. 111) also examined the joint effects of irrelevant sound and phonological similarity. Although this experiment has been cited as showing that irrelevant sound abolishes the effect of phonological similarity (Surprenant, Neath and LeCompte, 1999; Larsen et al., 2000; Baddeley, 2000), this is not actually what Jones and Macken found. In fact, they reported highly reliable effects of both phonological similarity and irrelevant sound, while "the interaction of list type and auditory condition was not significant" (Jones \& Macken, 1995, p. 111). To the extent to which there is any tendency for irrelevant sound and the phonological similarity to interact in their data, it is for the irrelevant sound effect to be smaller for phonologically similar lists, and not for irrelevant sound to eliminate the phonological similarity effect (see e.g. Figure 4, Jones \& Macken, p. 112). Note that Jones and Macken's experiment used seven-item lists, and their data are therefore completely consistent with Salamé and Baddeley's (1986) position that phonological similarity effects are to be expected when recall can be supported by the phonological loop.

At around the same time, Macken and Jones (1995) published data that they also interpreted as evidence that articulatory suppression and irrelevant sound are functionally equivalent. In a number of studies (Macken, Mosdell \& Jones, 1999; Tremblay, Macken and Jones, 2000; etc) Jones and colleagues have shown that the effect of irrelevant sound is greater for "changing-state" stimuli than for "steady-state" stimuli. For example, a series of different letters (changing-state) has a greater effect on recall than repetitions of a single letter (steady-state). Macken and Jones went further by showing that there is also a 
changing-state effect for mouthed articulatory suppression. Mouthing

repeatedly the letter name 'A' has less of an effect than mouthing the names of the letters A through to G. The changing-state effect for articulatory suppression was also found when the articulatory suppression was vocalized but masked by broadband noise presented through headphones. These effects of articulatory suppression were not, therefore, simply due to participants hearing themselves speak.

Macken and Jones' (1995) data are certainly interesting and, to the extent that they support an equivalence between irrelevant sound and articulatory suppression, are often considered challenging to a working memory perspective. We will, therefore, give two possible accounts of those data that reconcile them with both the other data described here and working memory theory more generally. The first, and in some ways simpler, account assumes that irrelevant sound can have its effect through the "inner ear" as through the "outer ear". To be explicit, the assumption would be that mouthed articulatory suppression would be recycled into the phonological loop in exactly the same way as subvocal (not mouthed) rehearsal is held to be. There, it would interfere with the contents of the store in a manner to be described below. In this way, changing-state articulation, even when only mouthed, would have the same effect as changing-state sound.

The second account is slightly more involved but has the twin advantages (from our perspective) that it not only leaves the working memory position intact but also accounts for one aspect of their data that presents difficulties for Macken and Jones's (1995) own interpretation. To understand this account, it should be noted that a critical feature of all four of Macken and Jones' 
experiments was that articulatory suppression took place only during a 10s retention interval between list presentation and recall. The question arises, therefore, as to what the participants were doing during the 10s retention interval. In an unfilled interval one would expect participants to have been engaged in subvocal rehearsal, consistent with the fact that such unfilled intervals do not typically lead to a steep drop in performance over time. Looking at the data, it seems to be highly likely that participants were also able to perform some rehearsal while performing "steady-state" suppression (repeatedly mouthing the letter name 'A'). Figure 1 replots the data from Macken and Jones' Experiment 5 (these are the data in their Figure 5, but arranged differently). It can be seen that, in the absence of changing-state irrelevant sound, there is only a 5\% drop in errors between the control condition and that involving 10 s of steady-state, mouthed articulatory suppression. This is nothing like the dramatic drop in performance that one would expect if rehearsal had been completely prevented throughout the retention interval (see e.g. Bjork \& Healy, 1974; Nairne \& Kelley, 1999; and Norris, Page \& Baddeley, submitted - all of whom used the much more demanding speeded digit-reading as a retention-interval task with dramatic effect on both performance and on the phonological similarity effect). Macken and Jones' participants were, therefore, almost certainly rehearsing under mouthed, steady-state articulatory suppression. If one accepts that rehearsal is likely to become progressively more difficult as one moves from a control task (tapping) to steady-state mouthed articulatory suppression, and more difficult still (perhaps even practically impossible) with changing-state, mouthed articulatory suppression, then one has no need to look any further for an explanation of Macken and Jones' data. There 
is indeed a changing-state effect for articulatory suppression, but the changing state manipulation does nothing more than alter the possibility of rehearsal.

Insert Figure 1 about here please

As noted earlier, such an assumption also explains one aspect of their own data with which Macken and Jones (1995) might otherwise have difficulty. Under conditions of external changing-state irrelevant sound, performance was further reduced, by about $9 \%$ relative to a tapping control, when subjects were required to perform steady-state, mouthed suppression (Experiment V; see Figure 1). However, if steady-state suppression is functionally equivalent to steady-state irrelevant sound, its effects should be weak; Jones and colleagues have repeatedly demonstrated that steady-state irrelevant sound has only a small effect. Moreover, Macken and Jones' own prediction with regard to Experiment $\mathrm{V}$ is that there should be a much reduced effect of both changing-state and steady-state mouthed suppression when there is a quite separate external source of changing-state irrelevant sound. So why is there quite a large effect of steady-state mouthed suppression under such conditions? Our explanation is that steady-state mouthed suppression has some effect on subjects' ability to rehearse, though the data strongly suggest that it is not sufficient to prevent rehearsal entirely.

In the context of all the above results, it is worth clarifying exactly how articulatory suppression (traditionally steady state) is assumed to influence memory (cf. Baddeley, 1986). First, it is assumed to prevent visually presented material being phonologically recoded and entering the phonological store. Second, articulatory suppression is assumed to block rehearsal during list 
presentation, even for auditory stimuli (though there is some question as to whether this blocking is complete, e.g. Baddeley, Lewis \& Vallar, 1984, p. 247). Last, when the burden of simultaneously processing incoming material is removed, steady-state articulatory suppression appears not to be able to prevent concurrent rehearsal of ordered material already in the phonological store. Other more demanding retention-interval tasks, including speeded digit-reading and possibly including changing-state suppression, are able to do so, as evidenced by their dramatic impact on performance. This summary might seem to be somewhat modified relative to the usual understanding of the working memory position, but in fact it is no more than a restatement of the conclusions of Vallar \& Baddeley (1982), who compared steady-state suppression, during a retention interval, with backwards counting - the latter proved much more disruptive to performance in a Peterson and Peterson (1959) task, suggesting that backward counting, but not steady-state articulatory suppression, was able to prevent rehearsal.

To conclude our review of relevant studies, we turn to Surprenant et al. (1999). In both order-reconstruction and ordered-recall tasks they found that, for visual but not auditory materials, irrelevant sound either eliminated, or greatly reduced, the phonological similarity effect. As Baddeley (2000) noted, this result is actually entirely consistent with Salamé and Baddeley's (1986) earlier work. Surprenant et al. used eight-item lists. Salamé and Baddeley also found that irrelevant sound abolished the phonological similarity effect with eight-item lists, though, crucially, not with shorter lists.

To summarize, a review of the relevant literature reveals a consistent picture across a variety of studies. For list lengths at or around span, there is no 
evidence that irrelevant sound abolishes the phonological similarity effect for visual materials. This contrasts strongly with the consistent effect of articulatory suppression, and casts doubt on any theory (such as the feature model — Neath, 2000; and O-OER — Macken \& Jones, 1995) that suggests that irrelevant sound and articulatory suppression are functionally equivalent.

Larsen and Baddeley (in press)

Larsen and Baddeley's (in press) contribution is to supply data that help to clarify still further the nature of irrelevant sound and articulatory suppression effects, and to place these results within an explicit, qualitative theoretical framework. They also weave into their account Saito's (1994) results with syncopated tapping and production. There are a number of points on which their data are clear. First, they confirm that, for six-item lists, articulatory suppression during list presentation and retention is sufficient to abolish the effect of phonological similarity. In contrast, phonological similarity effects persist under control, irrelevant sound and regular tapping conditions. As should be clear by now, this is absolutely consistent with comparable data to be found in the literature, and is clearly inconsistent with a functional equivalence between irrelevant sound and articulatory suppression. Second, the results are further support for Jones and colleagues' proposals regarding the importance of changing state within the irrelevant sound stimulus and, moreover, suggest that syncopation in the irrelevant stream is sufficient to represent a changing state. Third, Larsen and Baddeley support Saito's (1994) proposal that syncopated tapping abolishes the phonological similarity effect in a manner similar to articulatory suppression. Finally, Larsen and Baddeley 
show that even though the phonological similarity effect is preserved under irrelevant sound, for phonologically similar lists the effect of irrelevant sound is much reduced. It is to this issue that we now turn.

The tendency for the irrelevant sound effect to be reduced for phonologically similar lists is one that has been noted before (Jones and Macken, 1995; Salamé and Baddeley, 1986). As Baddeley (2000) reiterated, if recall is made difficult by using long lists, or phonologically confusable lists, subjects may abandon their use of the store. The work of Hanley and Bakopoulou (in press), to which Larsen and Baddeley refer, tends to support this interpretation. However, Larsen and Baddeley (in press) seem to go further than this and suggest, more generally, that "phonological similarity leaves the acoustic code severely impoverished and it is hence abandoned"( p.28 of MS). While this is a plausible explanation for their own study, where they found no effect of irrelevant sound for confusable lists, it departs from the more common working memory formulation in which the phonological similarity effect is viewed as a result of confusions within the store rather than due to the store's being abandoned in the face of confusable lists. If we accept their account, then the phonological similarity effect that they observe in the presence of irrelevant sound is not one based directly on the sensitivity of the phonological loop to the similarity manipulation.

One way to examine the effect of irrelevant sound on lists containing confusable items, while ensuring that any phonological similarity effect that is observed does not result from a listwise strategy change, involves using lists of mixed confusability. For such lists, the phonological similarity effect manifests itself as an increase in errors on confusable letters, with the remaining 
nonconfusables being recalled just as accurately as nonconfusable letters occupying corresponding positions in pure nonconfusable lists (Baddeley, 1968;

Henson, Norris, Page \& Baddeley, 1996; etc.). Such a manipulation can, therefore, give a measure of the phonological similarity effect within a single list, thus controlling for listwise strategy change. In an unpublished experiment, which factorially combined irrelevant sound and such a within-list measure of the phonological similarity effect, we have found strong effects of phonological confusability in the presence of both noise and irrelevant sound. This showed, once again, that a "standard" phonological similarity effect (i.e., one that cannot be attributed to a listwise strategy change) can be found in the presence of irrelevant sound. It was notable, however, that the effect of irrelevant sound on the phonologically confusable items in mixed lists was somewhat weaker than that seen for the nonconfusable items in the same lists. This does perhaps suggest that at least some subjects encounter problems when they come to recall the confusable items from the phonological loop, and at least sometimes, therefore, fall back, on an itemwise basis, on an alternative source that is insensitive to irrelevant sound.

Error analysis and modelling the effect of irrelevant sound

In the final section of this paper we will suggest how the irrelevant sound effect might be simulated within the primacy model of immediate serial recall (Page \& Norris, 1998). The primacy model might better be called a model of the phonological loop component of working memory, since that is the theoretical construct with which it is most clearly identified. For all of the effects that have previously been simulated within this quantitative model (and 
others e.g., Burgess \& Hitch, 1999; Henson, 1998; Brown, Preece, \& Hulme, 2000), including the phonological similarity effect, the word-length effect and the list-length effect, the modelling process has benefitted from the availability of detailed error analyses from the relevant experimental studies. With the kind cooperation of Jan Larsen, therefore, we have performed additional analyses on a subset of the data from Larsen and Baddeley (in press), so as to give us a clearer target for our simulations. Specifically, for the strong, changing-state irrelevant sound effect observed in Larsen and Baddeley's Experiment 3, we have derived serial position curves for both order errors and item errors according to Larsen (personal communication) the latter overwhelmingly comprise omissions though a small number of intrusions are also found.

The relevant serial position curves are shown in the upper panel of Figure 2. (For clarity, we have chosen to show both total errors and item errors, since order errors can trivially be derived as the difference between the two.) What is apparent is that the irrelevant sound effect is not only reflected in the number of order errors, but also in the number of item errors. A statistical analysis bears this out: we performed separate repeated-measures ANOVAs on the data from both order errors and item errors, in each case using the two factors, irrelevant sound (with two levels: present or absent) and serial position (with six levels). In the case of order errors, there were main effects of irrelevant sound, $\underline{\mathrm{F}}(1,23)=19.5, \underline{\mathrm{p}}<.001$, and serial position, $\underline{\mathrm{F}}(3.2,74.5)=13.9, \underline{\mathrm{p}}<.001$, with no interaction between the two, $\underline{\mathrm{F}}(2.9,67.3)=1.5$. (All F-tests are corrected for nonsphericity using the Greenhouse-Geisser method.) Similarly in the case of item errors, there were main effects of irrelevant sound, $\underline{\mathrm{F}}(1,23)=12.6, \underline{\mathrm{p}}=.002$, and serial position, 
$\underline{\mathrm{F}}(1.8,43.2)=6.9, \underline{\mathrm{p}}=0.003$, with no interaction between the two,

$\underline{\mathrm{F}}(2.6,60.8)=1.8$.

Insert Figure 2 about here please

These results show that Larsen and Baddeley's assumption (p. ??) that order errors rather than item errors are susceptible to irrelevant sound does not hold for their own data. Both order errors and item errors, principally omissions, increase with irrelevant sound.

So how might we simulate this pattern of errors in our primacy model? One quite simple way of modelling the disruption caused by irrelevant sound is in terms of an attentional resource. In our model, order across list items is stored as a primacy gradient of activation across localist representations of those list items. That is, the representations activate such that those corresponding to items presented earlier in the stimulus list have higher activations. The presence of another ordered stream, that is one whose changing state clearly indicates ordered material as opposed to repeated material, might cause the setting up of a second primacy gradient representing ordering within (a subsection of) that stream. Such a second primacy gradient would have to be streamed separately from that representing the to-be-remembered list to avoid direct interference. (In connectionist terms such binding might be carried out by placing the two different gradients in different phases of activation, though this is just one of many potential solutions.) It is reasonable to assume that the establishment of a second primacy gradient, albeit separately bound/streamed, will draw some resources away from the primacy gradient representing the to-be-remembered items. 
There are various ways in which this drop in resources might be operationalized in the model, but the data reviewed above, indicating an irrelevant sound effect on both order and item errors, strongly suggest that the best way of so doing is simply to "damp" the stimulus primacy gradient by multiplying the activations of which it comprises by a single number less than 1 . Qualitatively such damping will have two effects: first it will decrease the difference in activation between nodes representing list items, leading to more order errors; second, it will move all the activations closer to a given omission threshold, leading to more items errors. Before showing the results of quantitative simulations, it is worth noting that the suggested mechanism is also qualitatively consistent with four further observations.

First, our model is consistent with the observation that memory for (and indeed, perception of) between-stream order is notoriously poor (Bregman, 1990; etc.). In our suggested model, two ordered streams would be represented by different primacy gradients, which would be separately bound (e.g., in different activation phases) and whose relative levels overall would represent only the relative attentional resources devoted to the streams. Order within each stream would be represented by the individual primacy gradients themselves. Clearly, in such a framework, the ordering of items between streams cannot sensibly be derived from between-stream activation, consistent with the difficulty that such a task imposes.

Second, our model is consistent with the finding, now often replicated and still used in evidence against the working memory model, that the size of the irrelevant sound effect is not dependent on between-stream phonological similarity (references reviewed by Larsen and Baddeley, in press). In the 
primacy model, and in the development of it proposed here, order is stored at a level which is insensitive to phonological similarity. That is, the primacy gradient representing a list of confusables looks exactly like that representing a list of nonconfusables, other than being instated across a different set of localist item representations. If the effect of irrelevant sound represents the drop in resources caused by the presence of a second primacy gradient, it should be clear that this drop will not depend on the level of confusability within the irrelevant list. That level of confusability is in no way represented in the extent or character of its corresponding primacy gradient. This proposal should relieve the working memory model of the burden of the between-stream-similarity results which have previously been used to contradict it.

Third, our proposed mechanism is consistent with the importance of changing state to the size of the irrelevant sound effect. In our model, the effect comes about through a competition for resources between a primacy gradient representing order in the to-be-remembered list and another representing order in the irrelevant sound. To the extent that the irrelevant sound does not change state, it is unlikely to require resources at a level specifically designed for storing order in short-term memory. Put another way, it would make good sense for the system not to squander valuable order-representing resources on setting up a second primacy gradient to represent the unchanging irrelevant list "A A A A A...".

Fourth, our model of the irrelevant sound effect is qualitatively consistent with a 'dose' effect, as demonstrated by Bridges \& Jones (1996). A larger number of changes of state in an irrelevant stimulus should press its case for representation in ordered memory, that is, representation as a primacy gradient. 
One might think that such a secondary gradient becomes established with a certain probability at each change of state - the more changes in state, the more likely it is that the irrelevant stimulus comes to be represented at the order-storing level.

Note that our conception of dose effects and of changing-state effects is far from unique. Indeed, it bears a good deal of similarity to that of Jones and colleagues, whose careful experimental work has done much to characterize these effects. Where we have gone further is in developing these ideas in the context of an explicit quantitative model. Furthermore this is a model not just of the irrelevant sound effect, but of immediate serial recall in general.

All that remains is to attempt a quantitative simulation of the data presented in the upper panel of Figure 2. For this we used the standard five-parameter Primacy model described in Page and Norris (1998). (There is one additional parameter described in that paper. This parameter relates to phonological similarity, and is not relevant here.) We left all but one of the parameters at the values that had been used to simulate the Baddeley (1968) data in Page and Norris (1998). We changed only the parameter M (the standard deviation of the zero-mean Gaussian noise used in the threshold comparison stage, which influences omissions) from 0.74 to 0.85 . This single change in parameter values allowed to produce an excellent fit to total error curve from the Larsen and Baddeley's (in press) quiet control condition, together with a good fit to the item error curve, which nonetheless slightly underestimates the number of such errors particularly at early list positions. (This latter discrepancy is possibly because we are only modelling omission errors here and not the smaller number of additional intrusions). In order to 
model the effect of irrelevant sound we simply multiplied the primacy gradient activations by a new parameter with value less than 1, before commencing recall. A value of 0.67 gave again an excellent fit to the data from Larsen and Baddeley's irrelevant sound condition and in a way that reflects effects of irrelevant sound upon both order and item errors. The relevant graphs are shown in the lower panel of Figure 2.

To summarize the simulations, with a single additional parameter, and a change in value of only one of the parameters previously described (Page \& Norris, 1998, pp. 765-766), we have been able accurately to simulate the irrelevant sound effect on both order and item errors to be found in Larsen and Baddeley's third experiment. (We used the data from this experiment because it was the one in which they used standard changing-state materials and found their largest irrelevant sound effect.) No doubt, we could improve the precise quantitative fits by conducting a more complete search of the model's parameter space, but this seems superfluous given that we are already fitting the data to tolerances likely to be within those of experimental error. It should be remembered that the primacy model is a general, quantitative model of serial recall from phonological short-term memory, that has already been addressed to a large number of other phenomena (Page \& Norris, 1998). Other models like O-OER offer only a qualitative account of the irrelevant sound effect, in the absence of a viable general account of serial recall itself.

\section{Conclusion}

In this paper, we have reviewed literature on the irrelevant sound effect so as to highlight the considerable consistency that exists across studies. We have 
welcomed Larsen and Baddeley's (in press) contribution that buttresses the conclusion that irrelevant sound and articulatory suppression have different effects on short-term serial recall. We have alluded to some of our own data in further support, that showed, using a within-list measure, that the phonological similarity effect is not abolished by irrelevant sound but that suggest a weakening of the irrelevant sound effect for phonologically confusable items. We have extended Larsen and Baddeley's work by performing a more detailed error analysis that reveals how irrelevant sound affects both order errors and item errors, primarily omissions. Finally, we have described a development of our primacy model which captures both qualitative and quantitative aspects of the data on the irrelevant sound effect. 


\section{References}

Baddeley, A.D. (1968) How does acoustic similarity influence short-term memory? Quarterly Journal of Experimental Psychology, 20, 249-263.

Baddeley, A.D. (2000) The phonological loop and the irrelevant speech effect:

Some comments on Neath. Psychological Bulletin and Review, 7,544-549.

Baddeley, A.D., \& Hitch, G.J. (1974) Working memory. In G.H. Bower (Ed.), The psychology of learning and motivation, New York: Academic Press.

Baddeley, A.D., Lewis, V., \& Vallar, G. (1984) Exploring the articulatory loop. The Quarterly Journal of Experimental Psychology, 36A, 233-252.

Bjork,E.L., \& Healy, A.F. (1974) Short-term order and item retention. Journal of Verbal Learning and Verbal Behaviour, 13, 80-97.

Bregman, A.S. (1990) Auditory Scene Analysis. Cambridge, MA: MIT Press

Bridges, A.M., \& Jones, D.M. (1996) Word-dose in the disruption of serial recall by irrelevant speech: Phonological confusions or changing state? Quarterly Journal of Experimental Psychology, 49, 919-939.

Brown, G.D.A., Preece, T., \& Hulme, C. (2000) Oscillator-based memory for serial order. Psychological Review, 107, 127-181.

Burgess, N., \& Hitch, G.J. (1999) Memory for serial order: a network model of the phonological loop and its timing. Psychological Review, 106, 551-581.

Colle, H.A., \& Welsh, A. (1976) Acoustic masking in primary memory. Journal of Verbal Learning and Verbal Behaviour, 15, 17-31 
49-54.

Ellermeier, W., \& Hellbrück, J. (1998) Is level irrelevant in "irrelevant speech"?

Effects of loudness, signal-to-noise ratio, and binaural unmasking. Journal of Experimental Psychology: Human Perception and Performance, 24, 1406-1414.

Estes, W.K. (1973) Phonemic coding and rehearsal in short-term memory for letter strings. Journal of Verbal Learning and Verbal Behavior, 12, 360-372.

Hanley, J.R., \& Bakopoulou, E. (in press) Irrelevant speech, articulatory suppression and phonological similarity: A test of the phonological loop model and the feature model. Psychonomic Bulletin and Review.

Henson, R.N.A. (1998) Short-term memory for serial order: the start-end model. Cognitive Psychology, 36, 73-137.

Henson, R.N.A, Norris, D.G., Page, M.P.A., \& Baddeley, A.D. (1996) Unchained memory: Error patterns rule out chaining models of immediate serial recall. Quarterly Journal of Experimental Psychology, 49A(1), 80-115.

75-78. 21, 1008-1018.

Jones, D.M., \& Macken, W.J. (1995) Phonological similarity in the irrelevant speech effect. Within- or between-stream similarity? Journal of Experimental Psychology: Learning, Memory and Cognition, 21, 103-133.

Larsen, J.D., \& Baddeley, A.D. (in press) Disruption of verbal STM by irrelevant speech, articulatory suppression and manual tapping: Do they have a common source? Quarterly Journal of Experimental Psychology.

Larsen, J.D., Baddeley, A.D., \& Andrade, J. (2000) Phonological similarity and 
the irrelevant speech effect: Implications for models of short-term verbal memory. Memory,8, 145-158.

Levy, B.A. (1971) The role of articulation in auditory and visual short-term memory. Journal of Verbal Learning and Verbal Behavior, 10, 123-132.

Macken, W.J., \& Jones, D.M. (1995) Functional characteristics of the inner voice and inner ear: Single or double agency? Journal of Experimental Psychology: Learning, Memory and Cognition,21, 436-448.

Macken, W.J., Mosdell, N., \& Jones, D.M. (1999) Explaining the irrelevant-sound effect: Temporal distinctiveness or changing state? Journal of Experimental Psychology: Learning, Memory and Cognition,25, 1-6.

Murray, D.J. (1968) Articulation and acoustic confusability in short-term memory. Journal of Experimental Psychology, 78, 679-684.

Nairne, J.S., \& Kelley, M.R. (1999) Reversing the phonological similarity effect. Memory and Cognition, 27, 45-53.

Neath, I. (2000) Modeling the effects of irrelevant speech on memory. Psychonomic Bulletin and Review,7,403-423.

Norris, D., Page, M.P.A., \& Baddeley, A.D. (submitted) Retroactive effects of irrelevant speech on serial recall from short-term memory. Journal of Experimental Psychology: Learning, Memory \& Cognition.

Page, M., \& Norris, D. (1998) The primacy model. A new model of immediate serial recall. Psychological Review, 105, 761-781. 
Peterson, L.R., \& Peterson, M.J. (1959) Short-term retention of individual verbal items. Journal of Experimental Psychology, 58(3), 193-198.

Saito, S. (1994) What effect can rhythmic tapping have on the phonological similarity effect. Memory and Cognition, 22, 181-187.

Salamé, P., \& Baddeley, A.D. (1982) Disruption of short-term memory by unattended speech: Implications for the structure of working memory. Journal of Verbal Learning and Verbal Behaviour, 21, 150-164.

Salamé, P., \& Baddeley, A.D. (1986) Phonological factors in STM: Similarity and the unattended speech effect. Bulletin of the Psychonomic Society, 24, 263-265.

Salamé, P., \& Baddeley, A.D. (1987) Noise, unattended speech and short-term memory. Ergonomics, 30, 1185-1194.

Surprenant, A.M., Neath, I., \& and LeCompte, D.C. (1999) Irrelevant speech, phonological similarity, and presentation modality. Memory, 7, 405-420.

$181-191$.

Tremblay, S., Macken, W.J., \& Jones, D.M. (2000) Elimination of the word length effect by irrelevant sound revisited. Memory and Cognition, 28, 841-846. Vallar, G., \& Baddeley, A.D. (1982) Short-term forgetting and the articulatory loop. Quarterly Journal of Experimental Psychology, 34A, 53-60. 


\section{Author Notes}

Correspondence should be addressed to either of the authors. Mike Page is at the Dept. of Psychology, University of Hertfordshire, College Lane, Hatfield, AL10 9AB, U.K. (m.2.page@herts.ac.uk).Dennis Norris is at the MRC CBU, 15, Chaucer Rd., Cambridge, CB2 2EF, U.K. (dennis.norris@mrc-cbu.cam.ac.uk). 


\section{Figure Captions}

Figure 1. The results of Macken and Jones's (1995) Experiment 5 showing the effects of mouthed suppression either in quiet or in the presence of irrelevant sound.

Figure 2. Upper panel: Data showing the irrelevant sound effect found in Larsen \& Baddeley's (in press) Experiment 3. Lower panel: Simulations of these data using the primacy model (Page \& Norris, 1998). 

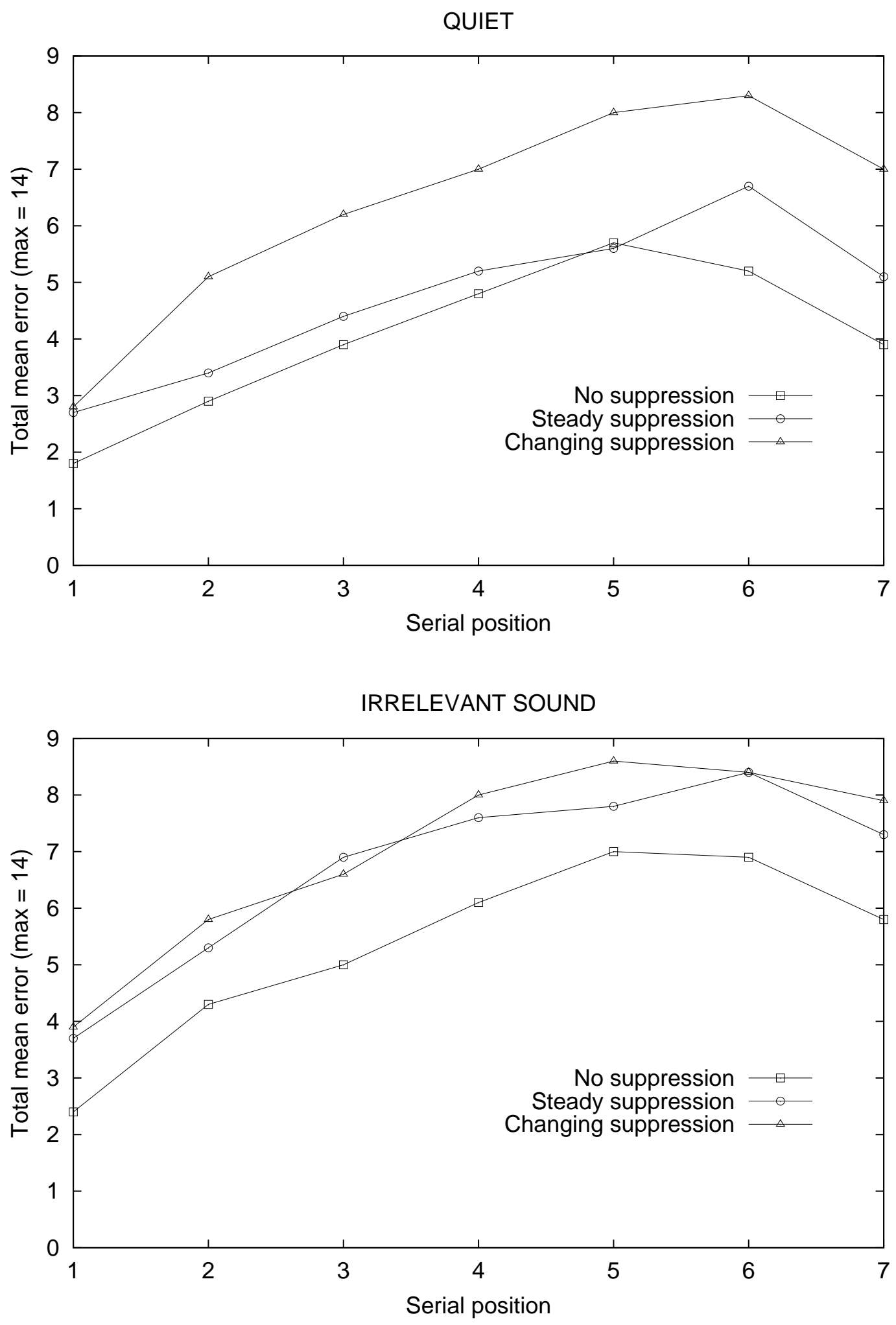

Figure 1: The results of Macken and Jones's (1995) Experiment 5 showing the effects of mouthed suppression either in quiet or in the presence of irrelevant sound. 

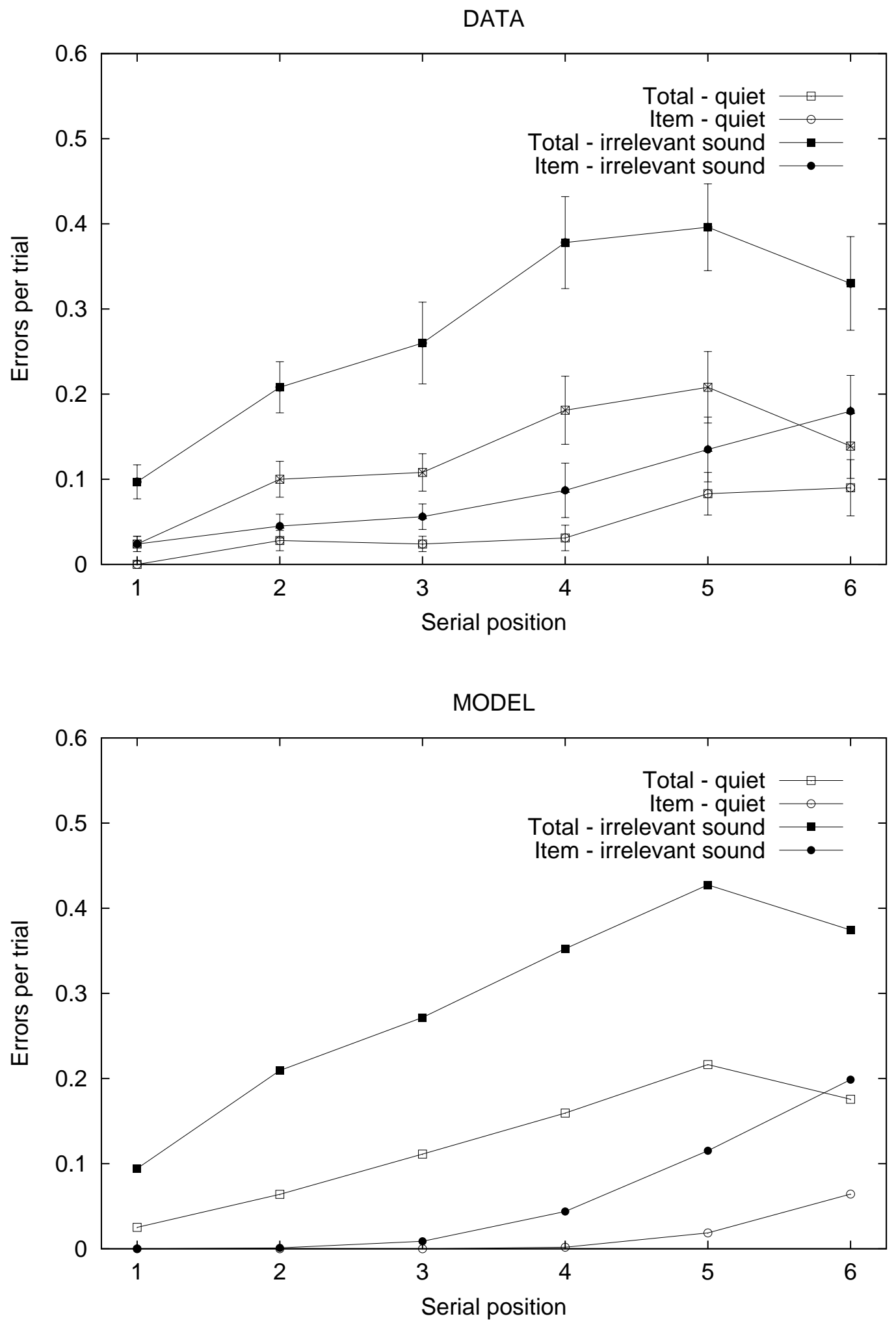

Figure 2: Upper panel: Data showing the irrelevant sound effect found in Larsen \& Baddeley's (in press) Experiment 3. Lower panel: Simulations of these data using the primacy model (Page \& Norris, 1998). 